\title{
GREEN SYNTHESIS OF PYRAZOLO [3,4]-PYRIMIDINE-THIONES USING IONIC LIQUID 2-METHYL-IMIDAZOLIUM-OXALATE AS POTENT EHRLICH ASCITES CARCINOMA RECEPTOR ANTAGONISTS
}

\author{
GANESH N YALLAPPA ${ }^{1}$, NAGARAJA $\mathrm{D}^{1 *}$, CHANDRASHEKHAR $\mathbf{U}^{2}$
}

${ }^{1}$ Department of PG Studies in Chemistry, Government Science College, Visvesvaraya Technological University-Research Resource Centre Chitradurga, Karnataka, India. ${ }^{2}$ Department of Chemistry, University B.D.T. College of Engineering, Visvesvaraya Technological University, Davangere, Karnataka, India. Email: nagarajachem.18@gmail.com

Received: 23 June 2019, Revised and Accepted: 05 August 2019

\section{ABSTRACT}

Objective: Pyrazolopyrimidines are heterocyclic molecules containing nitrogen as the main composition, and hence, they exhibit pharmacological efficacy. They are analogs of purines so that possessing wide applications in the field of medicinal chemistry. The main objective of this study is to synthesize different derivatives of pyrazole-pyrimidine classes by adopting simple methodology as well as by employing green chemistry. The purpose of the synthesis of these molecules is to study the antitumor activity against Ehrlich ascites carcinoma (EAC) cell lines.

Methods: After literature studies, it makes us to involve in the research of synthetic organic chemistry, especially to synthesize new compounds of pyrazolopyrimidines. We are reported solvent-free synthesis of pyrazolo [3,4- $d]$-pyrimidine-thiones through ethyl acetoacetate, hydrazine hydrate, thiourea, and different benzaldehydes. An ionic liquid 2-methyl-imidazolium-oxalate catalyzed the reactions under ultrasonication bath. Both conventional and ultrasonic methods were employed and comparison studies have been made. It was found that ultrasonic method completed the reaction quicker than the conventional method. All the synthesized compounds were confirmed their structures by ${ }^{1} \mathrm{HNMR}$, Fourier transform infrared, ${ }^{13} \mathrm{C}$-NMR, and elemental analysis spectra. The compounds were tested for in vitro anticancer activity against EAC cell lines. Most compounds revealed significant anticancer activity relative to doxorubicin as a positive control with inhibitory concentration $\left(\mathrm{IC}_{50}\right)$ values.

Results: Ultrasonication method is a simple method under which all the reactions were completed at faster time $(<7$ min) compared to the convention method. Among eight molecules, $\mathbf{8} \mathbf{a}$ and $\mathbf{8 d}$ completed the reactions at a faster rate. We reported $\mathrm{IC}_{50}$ values of all the molecules, in which $\mathbf{8 e}$ and $\mathbf{8 g}$ were exhibited excellent potency against EAC cell lines at different concentrations.

Conclusions: Ultrasonication method is an excellent method for the organic synthesis. We are herein reported that under this method, all the reactions are completed within $7 \mathrm{~min}$. Hence, it is superior method than the conventional method. All synthesized molecules have shown good inhibitor potency against EAC cell lines. Among them, two molecules $\mathbf{8 e}$ and $\mathbf{8} \mathbf{g}$ have shown excellent inhibitor potency.

Keywords: Ehrlich ascites carcinoma cell lines, Green chemistry, 2-methyl-imidazolium-hydroxide, Pyrazolo [3,4- $d$ ]-pyrimidine-thiones, Solvent free, Ultrasonicator method.

(C) 2019 The Authors. Published by Innovare Academic Sciences Pvt Ltd. This is an open access article under the CC BY license (http://creativecommons. org/licenses/by/4. 0/) DOI: http://dx.doi.org/10.22159/ajpcr.2019.v12i9.34661

\section{INTRODUCTION}

Pyrazolo $[3,4-d]$-pyrimidines are analogs of most purine-based drugs, having huge scope in the past decade for a consequence of their wide usage in medicinal field [1]. On literature studies, pyrazolopyrimidine derivatives have considerable potential in the field of chemotherapy, as they were found to exhibit their antitumor activity by inhibiting different types of enzymes such as cyclin-dependent kinase [2-4], Src and Abl tyrosine kinase [5], glycogen synthase kinase-3 [6-8], adenosine deaminase [9], and epidermal growth factor receptor protein tyrosine kinase. We synthesized pyrazolo $[3,4-d]$ - pyrimidinethiones through ethyl acetoacetate, hydrazine hydrate, thiourea, and different benzaldehydes by the ultrasonication under solvent-free condition. This methodology attained green chemistry. Reactions carried under ultrasonication were more effective for the reaction time and also for the high yield of the products than the conventional method [10]. 2-Mim ${ }^{+}$oxalate was used as a catalyst and found more efficient due to its ionic nature [11]. All the synthesized compounds were analyzed by Fourier transform infrared (FT-IR), ${ }^{1} \mathrm{HNMR}$, ${ }^{13} \mathrm{CNMR}$, and elemental analysis. The potency of these compounds was screened for in vitro test of anticancer activity against Ehrlich ascites carcinoma (EAC) cell lines. Most of the compounds showed excellent potency (inhibitory concentration $\left[\mathrm{IC}_{50}\right]$ values) against EAC cell lines.

\section{METHODS}

All chemicals were purchased by SDFCL Company. Reactions were managed by ultrasonic bath and magnetic stirrer (REMI). Melting point was determined by open capillary tubes in Buchi B-540 melting point apparatus. The reaction was monitored by thin-layer chromatography (TLC) using silica gel glass plates. FT-IR (Vertex version from Bruker), ${ }^{1} \mathrm{HNMR}$ (Bruker, $400 \mathrm{MHz}$ ), ${ }^{13} \mathrm{C}-\mathrm{NMR}$, and elemental analyzer were used. Biological activity (EAC cell lines) has been screened by Cytxon Biosolutions Pvt. Ltd., Hubballi - 580031, Karnataka, India.

3-(4,5-dimethylthiazol-2-yl)-2,5-diphenyltetrazolium bromide (MTT) assay protocol

The cells were trypsinized and aspirated into a $5 \mathrm{ml}$ centrifuge tube. Cell pellet was obtained by centrifugation at $300 \times$ g. The cell counted was adjusted, using Dulbecco's Modified Eagle's Medium HG medium, such that $200 \mu \mathrm{l}$ of suspension contained approximately 10,000 cells. To each well of the 96 -well microtiter plates, $200 \mu$ l of the cell suspension was added and the plate was incubated at $37^{\circ} \mathrm{C}$ and $5 \% \mathrm{CO}_{2}$ atmosphere for $24 \mathrm{~h}$. After $24 \mathrm{~h}$, the spent medium was aspirated. $200 \mu \mathrm{l}$ of different test concentrations $(100,200$, and $300 \mu \mathrm{g} / \mathrm{ml}$ from stock) of test drugs were added to the respective wells. The plate was then incubated at $37^{\circ} \mathrm{C}$ and $5 \% \mathrm{CO}_{2}$ atmosphere for $24 \mathrm{~h}$. The plate was removed from the incubator and the drug-containing media were aspirated. $200 \mu \mathrm{l}$ of 
media containing 10\% MTT reagent was then added to each well to get a final concentration of $0.5 \mathrm{mg} / \mathrm{ml}$ and the plate was incubated at $37^{\circ} \mathrm{C}$ and $5 \% \mathrm{CO}_{2}$ atmosphere for $3 \mathrm{~h}$

General procedure for the synthesis of pyrazolo $[3,4-d]$ pyrimidine-thiones

To a solution of ethyl acetoacetate $(10 \mathrm{mmol})$, add hydrazine hydrate (10 mmol), thiourea (10 mmol), and different benzaldehydes (10 $\mathrm{mmol})$ in a round bottom flask. The reaction mixture along with 2-methylimidazolium-oxalate was kept in an ultrasonic bath to proceed and monitored by TLC. The solid precipitate out in the solution and filtered off. The crude solid was recrystallized by hot water, ethanol, and finally dried.

\section{Preparation of 2-methyl-imidiazolium-oxalate}

2-methyl-imidazole $(10 \mathrm{mmol})$ was treated with sodium oxalate $(10 \mathrm{mmol})$ and dissolved in an acetone $(30 \mathrm{ml})$ solvent. The mixture was kept on magnetic stirrer for $30 \mathrm{~min}$. The reaction was monitored by TLC. The cleaned transparent solution was obtained.

\section{3-methyl-4-phenyl-1,3a-dihydro-6H-pyrazolo[3,4-d]pyrimidine-6- thione: (8a)}

Yellow crystals, m.p. $218-220^{\circ} \mathrm{C}$, yield $(81.00 \%)$. IR (KBr) $v \max /$ $\mathrm{cm}^{-1} 3343(\mathrm{NH}), 1652(\mathrm{C}=\mathrm{S}) .{ }^{1} \mathrm{H}-\mathrm{NMR}\left(\mathrm{CDCl}_{3}, 400 \mathrm{MHz}, \delta \mathrm{ppm}\right): 3.80$ (s, $\left.3 \mathrm{H}, \mathrm{OCH}_{3}\right), 6.82(\mathrm{~d}, 2 \mathrm{H}, \mathrm{J}=9.0 \mathrm{~Hz}, \mathrm{ArH}), 7.00(\mathrm{~d}, 1 \mathrm{H}, \mathrm{J}=4.8 \mathrm{~Hz}$, pyrimidine), $7.12(\mathrm{t}, 1 \mathrm{H}, \mathrm{ArH}), 7.33-7.46(\mathrm{~m}, 5 \mathrm{H}, \mathrm{ArH}), 7.62(\mathrm{~d}, 2 \mathrm{H}, \mathrm{J}=9.0 \mathrm{~Hz}, \mathrm{ArH})$, $7.54(\mathrm{~d}, 2 \mathrm{H}, \mathrm{J}=8.4 \mathrm{~Hz}, \operatorname{ArH}), 8.11$ (d, 2H, J=8.3 Hz, ArH), 8.48 (d, $1 \mathrm{H}$, $\mathrm{J}=4.8 \mathrm{~Hz}$, pyrimidine), $9.39(\mathrm{~s}, 1 \mathrm{H}, \mathrm{NH}), 10.05(\mathrm{~s}, 1 \mathrm{H}) .{ }^{13} \mathrm{C}-\mathrm{NMR}\left(\mathrm{CDCl}_{3}\right.$, $100 \mathrm{MHz}, \delta \mathrm{ppm}): 55.5\left(\mathrm{C}_{0} \mathrm{OCH}_{3}\right), 87.6\left(\mathrm{C}_{2} \mathrm{C}_{3}\right.$-Pyrazolopyrimidine $)$, 106.0 (C,C-Pyrazolopyrimidine), 114.7, 118.2, 120.8, 123.7, 126.7, 129.6, 129.8, 129.9(14C,Ar), 134.8 (C,C3a-pyrazolopyrimidine), 137.8, 142.8, 145.7 (3C,Ar), 147.7 (C,C-pyrazolopyrimidine), 163.2 $(\mathrm{C}=\mathrm{S})$.

Anal. calculated (\%) for $\mathrm{C}_{12} \mathrm{H}_{10} \mathrm{~N}_{4} \mathrm{~S}$ (242.00): C, 59.78; $\mathrm{H}, 4.26 ; \mathrm{N}, 23.28$, S, 12.68. Found: C, 59.69; H, 4.11; N, 23.00, S, 13.2\%.

\section{4-(2-chlorophenyl)-3-methyl-1,3a-dihydro-6H-Pyrazolo[3,4-d] pyrimidine-6-thione: $(8 \mathrm{~b})$}

Yellow crystals, m.p. $219-221^{\circ} \mathrm{C}$, yield(74.00\%). IR (KBr) vmax/ $\mathrm{cm}^{-1} 3334(\mathrm{NH}), 1648(\mathrm{C}=\mathrm{S}) .{ }^{1} \mathrm{H}-\mathrm{NMR}\left(\mathrm{CDCl}_{3^{\prime}}, 400 \mathrm{MHz}, \delta \mathrm{ppm}\right): 2.45$ $\left(\mathrm{s}, 3 \mathrm{H}, \mathrm{CH}_{3}\right), 3.6\left(\mathrm{~s}, 3 \mathrm{H}, \mathrm{OCH}_{3}\right), 6.77(\mathrm{~d}, 2 \mathrm{H}, \mathrm{J}=8.9 \mathrm{~Hz}, \mathrm{ArH}), 6.66(\mathrm{~d}$, $1 \mathrm{H}, \mathrm{J}=4.7 \mathrm{~Hz}), 7.06(\mathrm{t}, 1 \mathrm{H}, \mathrm{ArH}), 7.26(\mathrm{~d}, 2 \mathrm{H}, \mathrm{J}=8.3 \mathrm{~Hz}, \mathrm{ArH}), 7.28(\mathrm{t}$, $2 \mathrm{H}, \operatorname{ArH}), 7.56(\mathrm{~d}, 2 \mathrm{H}, \mathrm{J}=8.9 \mathrm{~Hz}, \operatorname{ArH}), 7.72$ (d, 2H, J=7.6 Hz, ArH), $8.10(\mathrm{~d}, 2 \mathrm{H}, \mathrm{J}=8.1 \mathrm{~Hz}, \mathrm{ArH}), 8.33$ (d, 1H, J=4.7Hz), $9.33(\mathrm{~s}, 1 \mathrm{H}, \mathrm{NH})$, $10.01(\mathrm{~s}, 1 \mathrm{H}, \mathrm{NH}) \cdot{ }^{13} \mathrm{C}-\mathrm{NMR}\left(\mathrm{CDCl}_{3}, 100 \mathrm{MHz}, \delta \mathrm{ppm}\right): 21.5\left(\mathrm{C} \mathrm{CH}_{3}\right)$, $55.6 \quad\left(\mathrm{C}, \mathrm{OCH}_{3}\right), 86.9$ (C,C3-pyrazolopyrimidine), $107.1 \quad\left(\mathrm{C}, \mathrm{C}_{6}-\right.$ pyrazolopyrimidine), 113.9, 119.1, 120.1, 123.5, 127.5, 129.0, 129.4, 129.6 (14C,Ar), 133.8 (C, $\mathrm{C}_{3 \mathrm{a}}$-pyrazolopyrimidine), 138.8, 142.2, 146.4 (3C, Ar), $163.1(\mathrm{C}=\mathrm{S})$.

Anal. calculated (\%) for $\mathrm{C}_{13} \mathrm{H}_{8} \mathrm{~N}_{4} \mathrm{OSCl}$ (303.00): C, 51.48; $\mathrm{H}, 2.64 ; \mathrm{N}$, 18.48; 0, 5.28; S, 10.56; Cl, 11.55. Found: C, 51.45H, 2.56; N, 18.56; O, 5.27; S, 10.55; Cl, $11.60 \%$.

\section{3-methyl-4-(2-methoxy-phenyl)-1,3a-dihydro-6H-pyrazolo[3,4-d] pyrimidine-6-thione: (8c)}

Yellow crystals, m.p. $206-208^{\circ} \mathrm{C}$, yield $(76.00 \%)$. IR (KBr) vmax/ $\mathrm{cm}^{-1} 3340(\mathrm{NH}), 1646$ (C=0). ${ }^{1} \mathrm{H}-\mathrm{NMR}\left(\mathrm{CDCl}_{3}, 400 \mathrm{MHz}, \delta \mathrm{ppm}\right): 3.80$ (s, $\left.3 \mathrm{H}, \mathrm{OCH}_{3}\right), 3.91\left(\mathrm{~s}, 3 \mathrm{H}, \mathrm{OCH}_{3}\right), 6.87(\mathrm{~d}, 2 \mathrm{H}, \mathrm{J}=8.9 \mathrm{~Hz}, \mathrm{ArH}), 6.89$ (d, $1 \mathrm{H}, \mathrm{J}=4.8 \mathrm{~Hz}), 7.05(\mathrm{~d}, 2 \mathrm{H}, \mathrm{J}=8.8 \mathrm{~Hz}, \mathrm{ArH}), 7.11(\mathrm{t}, 1 \mathrm{H}, \mathrm{ArH}), 7.37(\mathrm{t}, 2 \mathrm{H}$, $\mathrm{ArH}), 7.60(\mathrm{~d}, 2 \mathrm{H}, \mathrm{J}=8.9 \mathrm{~Hz}, \mathrm{ArH}), 7.72(\mathrm{~d}, 2 \mathrm{H}, \mathrm{J}=7.6 \mathrm{~Hz}, \mathrm{ArH}), 8.18(\mathrm{~d}, 2 \mathrm{H}$, $\mathrm{J}=8.8 \mathrm{~Hz}, \mathrm{ArH}), 8.40(\mathrm{~d}, 1 \mathrm{H}, \mathrm{J}=4.8 \mathrm{~Hz}), 9.36(\mathrm{~s}, 1 \mathrm{H}, \mathrm{NH}), 10.02(\mathrm{~s}, 1 \mathrm{H}, \mathrm{NH})$. ${ }^{13} \mathrm{C}$-NMR $\left(\mathrm{CDCl}_{3}, 100 \mathrm{MHz}, \delta \mathrm{ppm}\right): 55.5\left(\mathrm{C}, \mathrm{OCH}_{3}\right), 55.6\left(\mathrm{C}, \mathrm{OCH}_{3}\right), 87.4$ (C, $\mathrm{C}_{3}$-pyrazolopyrimidine), 106.4 (C, $\mathrm{C}_{6}$-pyrazolopyrimidine), 113.9, $114.2,119.0,120.0,122.4,123.5,128.9,131.3$ (14C,Ar), 134.0 (C, $\mathrm{C}_{3 a^{-}}$ pyrazolopyrimidine), 162.2 (C,Ar), $163.2(\mathrm{C}=0)$.

Anal. calculated (\%) for $\mathrm{C}_{13} \mathrm{H}_{12} \mathrm{~N}_{4} \mathrm{OS}$ (272.00): C, 57.35; $\mathrm{H}, 4.41 ; \mathrm{N}, 20.58$; 0, 5.88; S, 11.76. Found: C, 57.41; H, 4.45; N, 20.54; 0, 5.79; S. 11.97\%.
4-(2-hydroxyphenyl)-3-methyl-1,3a-dihydro-6H-pyrazolo[3,4-d] pyrimidine-6-thione: (8d)

Yellow crystals, m.p. $252-253^{\circ} \mathrm{C}$, yield(88.00\%); IR (KBr) vmax/ $\mathrm{cm}^{-1} 3348(\mathrm{NH}), 1641$ (C=S).1H-NMR ( $\left.\mathrm{CDCl}_{3}, 400 \mathrm{MHz}, \delta \mathrm{ppm}\right): 3.71$ (s, $\left.3 \mathrm{H}, \mathrm{OCH}_{3}\right), 6.78(\mathrm{~d}, 2 \mathrm{H}, \mathrm{J}=9.0 \mathrm{~Hz}, \mathrm{ArH}), 6.54(\mathrm{~d}, 1 \mathrm{H}, \mathrm{J}=4.7 \mathrm{~Hz}$, pyrimidine), $7.33(\mathrm{t}, 1 \mathrm{H}, \mathrm{ArH}), 7.35(\mathrm{t}, 2 \mathrm{H}, \mathrm{ArH}), 7.57(\mathrm{~d}, 4 \mathrm{H}, \mathrm{J}=8.8 \mathrm{~Hz}, \mathrm{ArH}), 7.77$ (d, $2 \mathrm{H}, \mathrm{J}=8.6 \mathrm{~Hz}, \mathrm{ArH}), 8.45(\mathrm{~d}, 2 \mathrm{H}, \mathrm{J}=8.7 \mathrm{~Hz}, \mathrm{ArH}), 8.52(\mathrm{~d}, 1 \mathrm{H}, \mathrm{J}=4.7 \mathrm{~Hz}$, pyrimidine), $9.44(\mathrm{~s}, 1 \mathrm{H}, \mathrm{NH}), 9.99(\mathrm{~s}, 1 \mathrm{H}, \mathrm{NH}) .{ }^{13} \mathrm{C}-\mathrm{NMR}\left(\mathrm{CDCl}_{3}, 100\right.$ $\mathrm{MHz}, \delta \mathrm{ppm}): 55.6\left(\mathrm{C}_{1} \mathrm{OCH}_{3}\right), 88.0\left(\mathrm{C}_{3} \mathrm{C}_{3}\right.$-pyrazolopyrimidine $), 107.0$ (C,C -pyrazolopyrimidine), 114.0, 119.2, 120.1, 123.7, 129.1, 129.1, 130.6, 131.8 (14C,Ar), $163.2(\mathrm{C}=\mathrm{S})$.

Anal. calculated (\%) for $\mathrm{C}_{12} \mathrm{H}_{10} \mathrm{~N}_{4} \mathrm{OS}(258.00)$ : C, 55.81; $\mathrm{H}, 3.87 ; \mathrm{N}, 21.70$; 0, 6.20; S, 12.40. Found: C, 55.74; H, 3.90; N, 21.85; 0, 6.22; S, 12.31\%.

3-methyl-4-(2-nitrophenyl)-1,3a-dihydro-6H-pyrazolo [3,4-d] pyrimidine-6-thione: (8e)

Yellow crystals, m.p. $278-280^{\circ} \mathrm{C}$, yield $(84.00 \%)$; IR (KBr) vmax/ $\mathrm{cm}^{-1} 3363(\mathrm{NH}), 1656$ (C=S). ${ }^{1} \mathrm{H}-\mathrm{NMR}$ (DMSO-d ${ }_{6}, 400 \mathrm{MHz}, \delta \mathrm{ppm}$ ): 3.71 $\left(\mathrm{s}, 3 \mathrm{H}, \mathrm{OCH}_{3}\right), 6.79(\mathrm{~d}, 2 \mathrm{H}, \mathrm{J}=9.0 \mathrm{~Hz}, \mathrm{ArH}), 7.22(\mathrm{t}, 1 \mathrm{H}, \mathrm{ArH}), 7.36(\mathrm{t}, 2 \mathrm{H}$, ArH), 7.45 (d, 1H, J=4.8 Hz), 7.56 (d, 2H, J=9.0 Hz, ArH), $7.71(d, 2 \mathrm{H}$, $\mathrm{J}=7.6 \mathrm{~Hz}, \mathrm{ArH}), 7.90(\mathrm{~d}, 2 \mathrm{H}, \mathrm{J}=8.7 \mathrm{~Hz}, \mathrm{ArH}), 8.21(\mathrm{~d}, 2 \mathrm{H}, \mathrm{J}=8.7 \mathrm{~Hz}, \mathrm{ArH}), 8.72$ (d, $1 \mathrm{H}, \mathrm{J}=4.8 \mathrm{~Hz}), 9.28(\mathrm{~s}, 1 \mathrm{H}, \mathrm{NH}), 10.04(\mathrm{~s}, 1 \mathrm{H}, \mathrm{NH}) \cdot{ }^{13} \mathrm{C}-\mathrm{NMR}$ (DMSO-d $\mathrm{d}_{6}$ $100 \mathrm{MHz}, \delta \mathrm{ppm}): 55.7\left(\mathrm{C}, \mathrm{OCH}_{3}\right), 87.6$ (C, $\mathrm{C}_{3}$-pyrazolopyrimidine), 106.9 (C,C -pyrazolopyrimidine), 114.4, 119.1, 120.5, 123.3, 129.4, 129.8, 131.0, 131.6 (14C,Ar), 133.7 (C, C $\mathrm{C}_{3 \mathrm{a}}$-pyrazolopyrimidine), 163.7 (C=S).

Anal. calculated $\left(\mathrm{C}_{12} \mathrm{H}_{9} \mathrm{~N}_{5} \mathrm{O}_{2} \mathrm{~S}, \%\right.$ ) for (287.00): C, 50.17, H, 3.13; N, 24.39, O, 11.15, S, 11.15. Found: C, 50.45; H, 3.05; N, 24.34, O, 11.01, S, $11.21 \%$.

4-(2,4-dinitrophenyl)-3-methyl-1,3a-dihydro-6H-pyrazolo[3,4-d] pyrimidine-6-thione: (8f)

Yellow crystals, m.p. $237-239^{\circ} \mathrm{C}$, yield $(67.00 \%)$; IR (KBr) vmax/ $\mathrm{cm}^{-1} 3341$ (NH), 1649 (C=S). ${ }^{1} \mathrm{H}-\mathrm{NMR}$ (DMSO-d, $400 \mathrm{MHz}, \delta \mathrm{ppm}$ ): $3.71\left(\mathrm{~s}, 3 \mathrm{H}, \mathrm{OCH}_{3}\right), 6.95(\mathrm{~d}, 2 \mathrm{H}, \mathrm{J}=9.0 \mathrm{~Hz}, \mathrm{ArH}), 7.16(\mathrm{t}, 1 \mathrm{H}, \mathrm{ArH})$, 7.38 (d, 1H, J=4.9 Hz), 7.35 (d, 2H, J=7.6 Hz, ArH), 7.52 (t, 2H, ArH), $7.54(\mathrm{~d}, 2 \mathrm{H}, \mathrm{J}=9.0 \mathrm{~Hz}, \mathrm{ArH}), 7.79$ (d, 2H, J=8.6 Hz, ArH), 8.35 (d, $2 \mathrm{H}$, $\mathrm{J}=8.9 \mathrm{~Hz}, \mathrm{ArH}), 8.79(\mathrm{~d}, 1 \mathrm{H}, \mathrm{J}=4.8 \mathrm{~Hz}), 9.33(\mathrm{~s}, 1 \mathrm{H}, \mathrm{NH}), 10.05(\mathrm{~s}, 1 \mathrm{H}$,

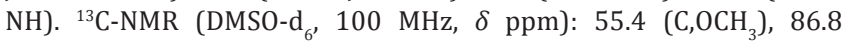

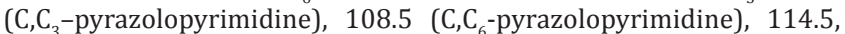
115.8, 115.9, 118.7, 119.5, 123.6, 126.5, 129.1 (14C, Ar), 132.3 (C, $\mathrm{C}_{3 \mathrm{a}}{ }^{-}$ pyrazolopyrimidine), $162.2(\mathrm{C}=\mathrm{S})$.

Anal. calculated (\%) for $\left(\mathrm{C}_{12} \mathrm{H}_{8} \mathrm{~N}_{6} \mathrm{O}_{4} \mathrm{~S}, \%\right)$ for (332.00): C, 43.37, $\mathrm{H}, 2.40$; N, 25.30, 0, 19.27, S, 9.63. Found: C, 43.45; H, 2.57; N, 25.50, 0, 19.58, S, $8.90 \%$.

4-(2,4-dihydroxyphenyl)-3-methyl-1,3a-dihydro-6H-pyrazolo[3,4- $d$ ] pyrimidine-6-thione: (8g)

Yellow crystals, m.p. $233-235^{\circ} \mathrm{C}$, yield(76.00\%); IR (KBr) vmax/ $\mathrm{cm}^{-1} 3351(\mathrm{NH}), 1658$ (C=S). ${ }^{1} \mathrm{H}-\mathrm{NMR}$ (DMSO-d $\left.{ }_{6}, 400 \mathrm{MHz}, \delta \mathrm{ppm}\right): 3.76$ $\left(\mathrm{s}, 3 \mathrm{H}, \mathrm{OCH}_{3}\right), 7.05(\mathrm{~d}, 2 \mathrm{H}, \mathrm{J}=8.4 \mathrm{~Hz}, \mathrm{ArH}), 7.22(\mathrm{t}, 1 \mathrm{H}, \mathrm{ArH}), 7.42(\mathrm{t}, 2 \mathrm{H}$, $\operatorname{ArH}), 7.47(\mathrm{t}, 1 \mathrm{H}, \mathrm{J}=4.9 \mathrm{~Hz}), 7.74(\mathrm{~d}, 2 \mathrm{H}, \mathrm{J}=7.8 \mathrm{~Hz}, \mathrm{ArH}), 7.82(\mathrm{~d}, 2 \mathrm{H}$, $\mathrm{J}=8.5 \mathrm{~Hz}, \mathrm{ArH}$ ), 7.91 (d, 1H, J=4.6 Hz), 8.29 (d, 1H, J=4.4 Hz), 8.58 (d, $1 \mathrm{H}, \mathrm{J}=2.8 \mathrm{~Hz}), 8.71(\mathrm{~d}, 1 \mathrm{H}, \mathrm{J}=4.4 \mathrm{~Hz}$, pyrimidine), $9.44(\mathrm{~s}, 1 \mathrm{H}, \mathrm{NH}), 10.07$ (s, $1 \mathrm{H}, \mathrm{NH}$ ). ${ }^{13} \mathrm{C}-\mathrm{NMR}$ (DMSO-d $, 100 \mathrm{MHz}, \delta \mathrm{ppm}$ ): $55.7\left(\mathrm{C}_{6} \mathrm{OCH}_{3}\right.$ ), 86.8 (C, $\mathrm{C}_{3}$-pyrazolopyrimidine), 107.3 (C, $\mathrm{C}_{6}$-pyrazolopyrimidine), 114.8, 119.2, 120.8, 126.3(7C, Ar), 128.1, 129.8(2C), 130.1(2C,Ar), 162.9 $(\mathrm{C}=\mathrm{S})$.

Anal. calculated (\%) for $\mathrm{C}_{12} \mathrm{H}_{10} \mathrm{~N}_{4} \mathrm{O}_{2} \mathrm{~S}(274.00)$ : C, 52.55; H, 3.64; N, $20.43,0,11.67$, S, 11.67. Found: C, 52.35; H, 3.70; N, 20.71; 0, 11.51; S, $11.730 \%$.

4-(2,4-dimethylphenyl)-3-methyl-1,3a-dihydro-6H-pyrazolo[3,4-d] pyrimidine-6-thione: $(\mathbf{8 h})$

Yellow crystals, m.p. $251-253^{\circ} \mathrm{C}$, yield $(75.00 \%)$; IR $(\mathrm{KBr})$ vmax/ $\mathrm{cm}^{-1} 3372(\mathrm{NH}), 1662$ (C=S);1H-NMR ( $\mathrm{CDCl}_{3}, 400 \mathrm{MHz}, \delta \mathrm{ppm}$ ): 2.35 (s, 3H, CH3), $3.80(\mathrm{~s}, 3 \mathrm{H}, \mathrm{OCH} 3), 6.88(\mathrm{~d}, 2 \mathrm{H}, \mathrm{J}=9.0 \mathrm{~Hz}, \mathrm{ArH}), 6.95(\mathrm{~d}$, $1 \mathrm{H}, \mathrm{J}=4.8 \mathrm{~Hz}$, pyrimidine), $7.18(\mathrm{~d}, 2 \mathrm{H}, \mathrm{J}=8.2 \mathrm{~Hz}, \mathrm{ArH}), 7.40(\mathrm{~d}, 2 \mathrm{H}$, 
J=8.2 Hz, ArH), 7.60-7.64 (m, 5H, ArH), 8.10 (d, $2 \mathrm{H}, \mathrm{J}=8.2 \mathrm{~Hz}, \mathrm{ArH})$, 8.45 (d, 1H, J=4.8 Hz,), $9.41(\mathrm{~s}, 1 \mathrm{H}, \mathrm{NH}), 9.95(\mathrm{~s}, 1 \mathrm{H}, \mathrm{NH}) .{ }^{13} \mathrm{C}-\mathrm{NMR}$ $\left(\mathrm{CDCl}_{3}, 100 \mathrm{MHz}, \delta \mathrm{ppm}\right): 21.1$ (C,CH3), 55.5 (C,OCH3), 87.5 (C,C $\mathrm{C}_{3}-$ pyrazolopyrimidine), 106.8 (C, $\mathrm{C}_{6}$-pyrazolopyrimidine), 114.4, 119.2, 120.1, 127.3, 129.3, 129.7, 133.7 (14C, Ar), 134.2 (С, $\mathrm{C}_{3 а}{ }^{-}$ pyrazolopyrimidine), $163.2(\mathrm{C}=0)$.

Anal. calculated (\%) for $\mathrm{C}_{14} \mathrm{H}_{14} \mathrm{~N}_{4} \mathrm{~S}(270.00)$ ): C, 62.22; H, 5.16; N, 20.74; S, 11.85. Found: C, 62.20; H, 5.11; N, 20.79; S, 11.90\%.

\section{RESULTS AND DISCUSSION}

All synthesized molecules are confirmed by FT-IR, 3300-3400 $\mathrm{cm}^{-1}$ ( $\mathrm{NH}$ group of pyrazole) and $1630-1660 \mathrm{~cm}^{-1}(\mathrm{C}=\mathrm{S}),{ }^{1} \mathrm{HNMR}$ by $6.7 \mathrm{ppm}-8.5 \mathrm{ppm}$ confirms aromatic hydrogen, and 8.5-10.1 indicated the presence of - $\mathrm{NH}$ protons. To follow the green chemistry, solventfree reactions were maintained under ultrasonication Reaction Scheme-1 [12]. Ionic liquids found to be better catalysts for organic synthesis. Bronsted acidic ionic liquid 2-methyl-imidazolium-oxalate catalyzed the reactions for good yield as well as instant completion. Experimental data revealed that ultrasonic method is a more superior method than conventional. Reaction times and yield of the products were studied for both methods (Table 1) [13].
The conventional method was managed at room temperature by stirring. Under this method, the reaction times for all the molecules are higher and yields were lower than ultrasonication method.

From Table 1, under ultrasonication, we found that $\mathbf{8 a}$ and $\mathbf{8 d}$ molecules completed the reaction at shortest time and yielded the highest quantity (\%). Due to electron releasing group present at the 2-position of phenyl substituent, both $\mathbf{8 a}$ and $\mathbf{8 d}$ are found to be stable molecules. Hence, these molecules yield excellent. Similarly, 8e, $\mathbf{8 f}$, and $\mathbf{8 g}$ molecules possess electron-withdrawing group at phenyl substituent and, therefore, yield lower than the molecules possessing electron releasing group.

Under the convention method, $\mathbf{8 c}$ and $\mathbf{8 d}$ compounds ended the reactions at shortest time. $\mathbf{8 e}$ and $\mathbf{8 f}$ molecules yield lower than the remaining molecules due to the presence of electron-withdrawing group at phenyl substituent.

The preliminary investigation of the anticancer activity of the newly synthesized pyrazolopyrimidine derivatives was screened in vitro against EAC. The $\mathrm{IC}_{50}$ values of these molecules were determined (Tables 2-4) [14-17]. From the data, we concluded that at $100 \mu \mathrm{g} / \mathrm{ml}$,

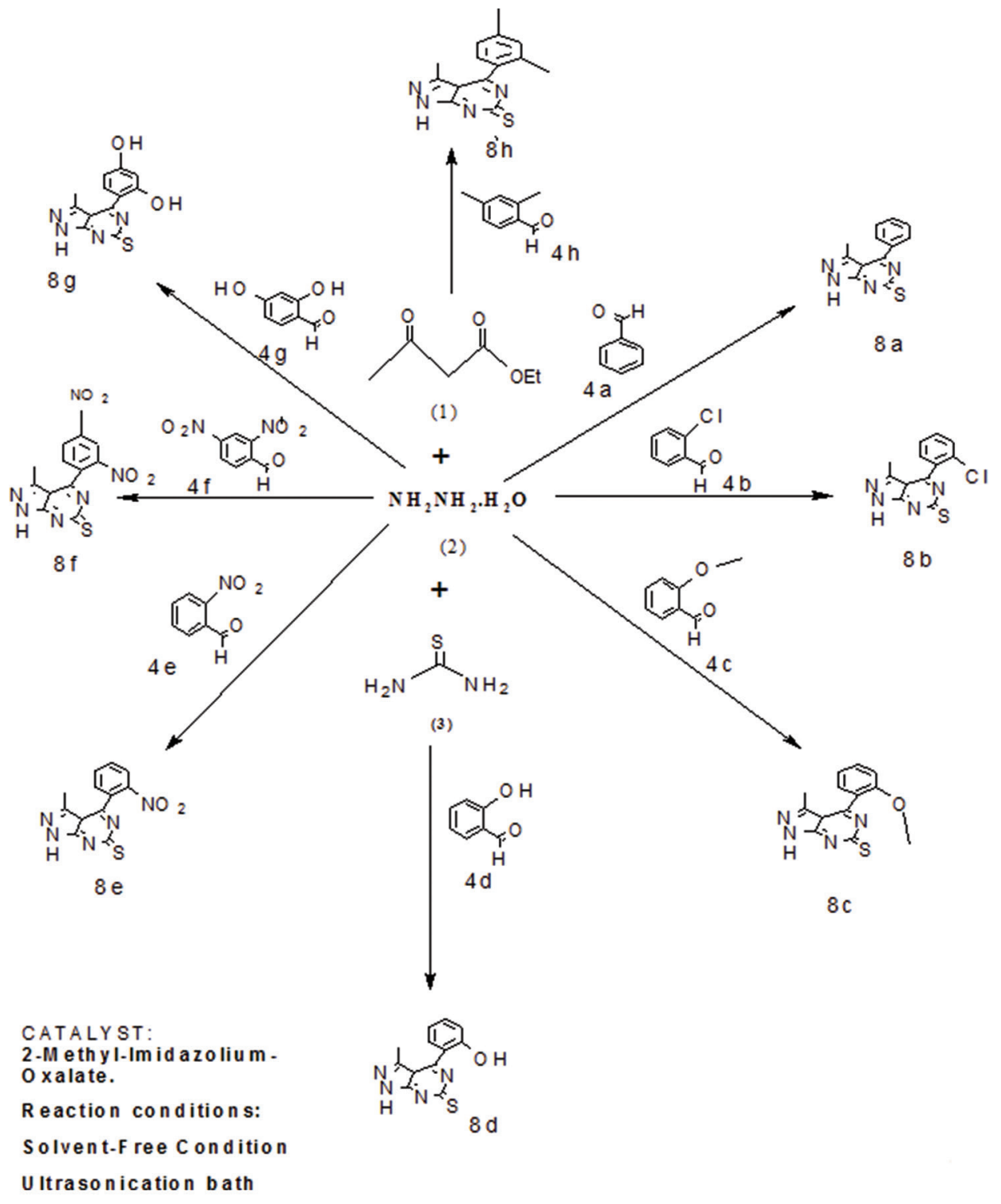

Reaction Scheme-1: Schematic representation for the synthesis of pyrazolo-pyrimidine-thiones (8a-8h) 
the higher anticancer activity of molecules $\mathbf{8 e}$ and $\mathbf{8 g}$ (Table 1 and Fig. 1) is attributed to the presence of nitro group (2-position) and two $\mathrm{OH}$ groups in addition to $\mathrm{C}=\mathrm{S}$ group that facilitates the $\mathrm{C}-\mathrm{S}$ bonding with the active sites which increase its reactivity respectively.

From Fig. 2, at $200 \mu \mathrm{g} / \mathrm{ml}, \mathbf{8 e}$ and $\mathbf{8 g}$ molecules have excellent $\mathrm{IC}_{50}$ values.

Table 1: Comparison data for the compounds $b / w$ conventional and ultrasonicator methods

\begin{tabular}{lllll}
\hline Compounds & $\begin{array}{l}\text { Conventional } \\
\text { method at RT } \\
\text { time (min) }\end{array}$ & $\begin{array}{l}\text { Yield } \\
\mathbf{( \% )}\end{array}$ & $\begin{array}{l}\text { Ultrasonic } \\
\text { method at } \\
\mathbf{8 0}^{\circ} \text { C time (min) }\end{array}$ & $\begin{array}{l}\text { Yield } \\
\text { (\%) }\end{array}$ \\
\hline $\mathbf{8 a}$ & 10 & 79.00 & 2 & 81.00 \\
$\mathbf{8 b}$ & 12 & 76.00 & 3 & 74.00 \\
$\mathbf{8 c}$ & 8 & 68.00 & 4.5 & 76.00 \\
$\mathbf{8 d}$ & 10 & 78.00 & 2 & 88.00 \\
$\mathbf{8 e}$ & 14 & 63.00 & 4 & 84.00 \\
$\mathbf{8 f}$ & 11 & 69.00 & 6 & 67.00 \\
$\mathbf{8 g}$ & 14 & 74.00 & 3 & 76.00 \\
$\mathbf{8 h}$ & 12 & 73.00 & 7 & 75.00 \\
\hline
\end{tabular}

Table 2: Binding energy and $\mathrm{IC}_{50}$ values of compounds $(100 \mu \mathrm{g} / \mathrm{ml})$ against EAC

\begin{tabular}{lll}
\hline Tested compounds & IC $_{50}$ values in $\boldsymbol{\mu g} / \mathbf{m l}$ & Binding energy \\
\hline $\mathbf{8 a}$ & 84 & 51.21 \\
$\mathbf{8 b}$ & 151 & 54.92 \\
$\mathbf{8 c}$ & 123 & 51.77 \\
$\mathbf{8 d}$ & 108 & 52.11 \\
$\mathbf{8 e}$ & 49 & 48.39 \\
$\mathbf{8 f}$ & 185 & 59.90 \\
$\mathbf{8 g}$ & 79 & 54.33 \\
$\mathbf{8 h}$ & 94 & 57.89 \\
Doxorubicin & 59 & 46.73 \\
\hline
\end{tabular}

EAC: Ehrlich ascites carcinoma, $\mathrm{IC}_{50}:$ Inhibitory concentration

Table 3: Binding energy and $\mathrm{IC}_{50}$ values of compounds $(200 \mu \mathrm{g} / \mathrm{ml})$ against EAC

\begin{tabular}{lll}
\hline Tested compounds & IC $_{50}$ values in $\boldsymbol{\mu g} / \mathbf{m l}$ & Binding energy \\
\hline $\mathbf{8 a}$ & 86 & 54.33 \\
$\mathbf{8 b}$ & 123 & 51.12 \\
$\mathbf{8 c}$ & 78 & 48.72 \\
$\mathbf{8 d}$ & 185 & 57.53 \\
$\mathbf{8 e}$ & 66 & 42.43 \\
$\mathbf{8 f}$ & 211 & 51.81 \\
$\mathbf{8 g}$ & 35 & 54.11 \\
$\mathbf{8 h}$ & 144 & 56.99 \\
Doxorubicin & 76 & 47.88 \\
\hline
\end{tabular}

EAC: Ehrlich ascites carcinoma, $\mathrm{IC}_{50}$ : Inhibitory concentration

Table 4: Binding energy and $\mathrm{IC}_{50}$ values of compounds $(300 \mu \mathrm{g} / \mathrm{ml})$ against EAC

\begin{tabular}{lll}
\hline Tested compounds & IC $_{\mathbf{5 0}}(\boldsymbol{\mu g} / \mathbf{m l})$ EAC & Binding energy \\
\hline $\mathbf{8 a}$ & 85 & 54.62 \\
$\mathbf{8 b}$ & 110 & 52.51 \\
$\mathbf{8 c}$ & 111 & 49.78 \\
$\mathbf{8 d}$ & 102 & 56.34 \\
$\mathbf{8 e}$ & 46 & 46.77 \\
$\mathbf{8 f}$ & 157 & 57.74 \\
$\mathbf{8 g}$ & 68 & 52.35 \\
$\mathbf{8 h}$ & 79 & 53.31 \\
Doxorubicin & 53 & 49.99 \\
\hline
\end{tabular}

EAC: Ehrlich ascites carcinoma, $\mathrm{IC}_{50}$ : Inhibitory concentration
From Fig. 3, at $300 \mu \mathrm{g} / \mathrm{ml}, \mathbf{8 e}$ and $\mathbf{8 g}$ also possess excellent $\mathrm{IC}_{50}$ values.

It is competing with the substrate, so the concentration of ligand needed to reduce the enzymatic activity (EAC) by $50 \%$ depends on the concentration of substrate and how tightly it binds the enzyme. The experimental binding energies $(\Delta \mathrm{Gexp})$ were calculated from the measured $\mathrm{IC}_{50}$ by Equation (1), using the gas constant (R) and the temperature $(\mathrm{T})$ [18-20].

$\Delta \mathrm{Gexp}=-\mathrm{RT} \ln \mathrm{IC}_{50}$

Where, " $\Delta G \exp$ " is the binding energy of the molecule with cancer cell lines,

" $\mathrm{R}$ " is the universal gas constant $\left(8.314 \mathrm{~J} \mathrm{~K}^{-1} \mathrm{~mol}^{-1}\right)$,

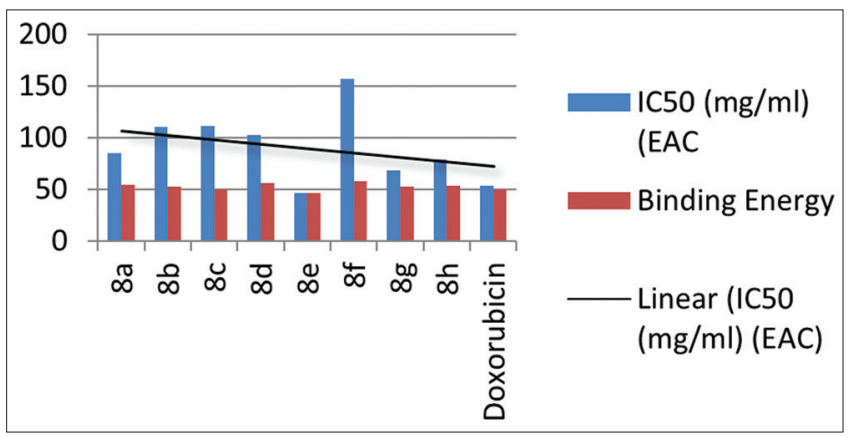

Fig. 1: Graphical representation of compounds $(100 \mu \mathrm{g} / \mathrm{ml})$ against inhibitory concentration 50 values of Ehrlich ascites carcinoma cell lines

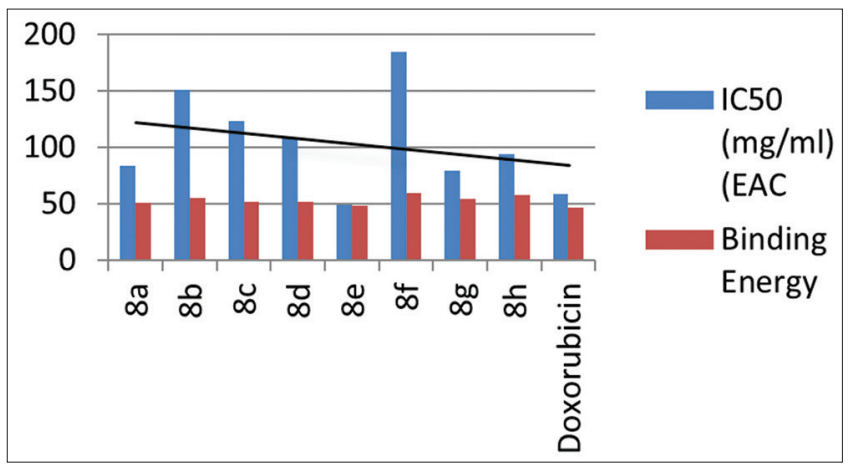

Fig. 2: Graphical representation of compounds $(200 \mu \mathrm{g} / \mathrm{ml})$ against inhibitory concentration 50 values of Ehrlich ascites carcinoma cell lines

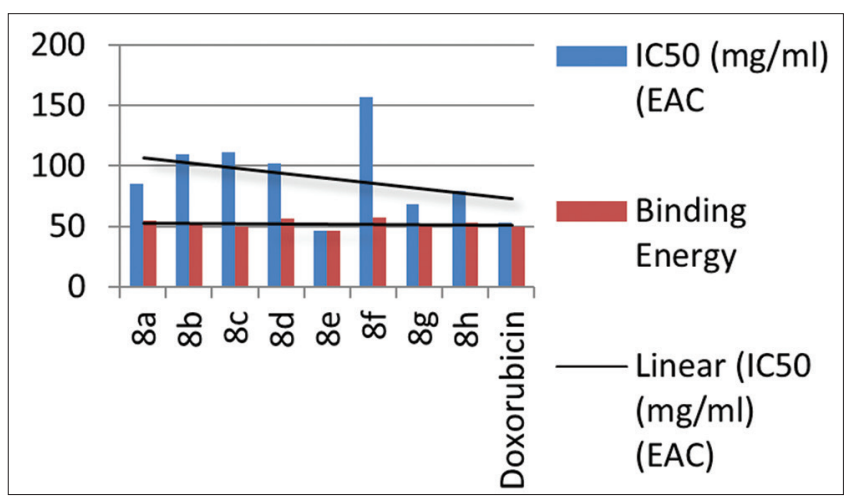

Fig. 3: Graphical representation of compounds $(300 \mu \mathrm{g} / \mathrm{ml})$ against inhibitory concentration 50 values of Ehrlich ascites carcinoma cell lines 
"T" is the temperature at which a molecule binds $\left(298^{\circ} \mathrm{K}\right)$ at room temperature,

"IC ${ }_{50}$ " is the inhibitory concentration of the molecule.

\section{Results and interpretation}

The $\mathrm{IC}_{50}$ values of the test compounds for EAC cell line for $24 \mathrm{~h}$ treatment were found to be

\begin{tabular}{|c|c|}
\hline $\begin{array}{l}\text { Sample } \\
\text { name }\end{array}$ & $\begin{array}{l}\text { Ehrlich ascites carcinoma cell line inhibitory } \\
\text { concentration } 50 \text { (in } \mu \mathrm{g} / \mathrm{ml}) 24 \mathrm{~h}\end{array}$ \\
\hline Doxorubicin & 15 \\
\hline **Sample 8a & $<100$ \\
\hline Sample 8b & $>100$ \\
\hline \multirow[t]{2}{*}{ **Sample 8c } & $>100.00$ \\
\hline & Calculated value: 88.5 \\
\hline \multirow[t]{2}{*}{ **Sample 8d } & $>100.00$ \\
\hline & Calculated value: 149.71 \\
\hline Sample 8e & $<100.00$ \\
\hline Sample $8 f$ & $>150.00$ \\
\hline Sample 8g & $<100.00$ \\
\hline Sample $8 \mathrm{~h}$ & $<100.00$ \\
\hline
\end{tabular}

\section{CONCLUSIONS}

We reported that organic reactions were brought very good yield by momentarily under ultrasonic waves compared to the conventional method. 2-methyl-imidazolium-oxalate, an ionic liquid was found to be a good catalyst for organic syntheses. Pyrazolopyrimidines proved as potent molecules for cancer treatment. All the synthesized molecules exhibited moderate inhibition against EAC cell lines. $\mathbf{8 e}$ and $\mathbf{8 g}$ have the most potent $\mathrm{IC}_{50}$ values.

\section{ACKNOWLEDGMENTS}

One of the authors, Ganesh N Yallappa acknowledges the Other Backward Classes Commission, Government of Karnataka, for providing a Research Fellowship during this period.

\section{CONFLICTS OF INTEREST}

Authors declare that they have no conflicts of interest.

\section{AUTHORS' CONTRIBUTIONS}

All the authors have contributed equally in the design, development, review, and finalization of the contents of the manuscript.

\section{REFERENCES}

1. Abd El Razik HA, Abdel Wahab AE. Synthesis and biological evaluation of some novel fused pyrazolo-pyrimidines as potential anticancer and antimicrobial agents. Arch Pharm Chem Life Sci 2011;11:184-96.

2. Aggarwal R, Masan E, Kaushik P, Kaushik D, Sharma C, Aneja KR. Synthesis and biological evaluation of 7-trifluoromethylpyrazolo[1,5-a] pyrimidines as anti-inflammatory and antimicrobial agents. J Fluor Chem 2014;168:16-24
3. Kaping S, Kalita U, Sunn M, Singha LI., Vishwakarma JN. A facile, regioselective synthesis of pyrazolo[1,5-a]pyrimidine analogs in the presence of $\mathrm{KHSO}_{4}$ in aqueous media assisted by ultrasound and their anti-inflammatory and anti-cancer activities. Monatsh Chem 2016;147:1257-76

4. Deshmukh S, Dingore K, Gaikwad V, Jachak M. An efficient synthesis of pyrazolo[1,5-a]pyrimidines and evaluation of their antimicrobial activity. J Chem Sci 2016;128:1459-68.

5. El-Mekabaty A, Habib OM, Moawad EB, Hasel A. Synthesis and antioxidant activity of new pyrazolo[1,5-a]pyrimidine derivatives incorporating a thiazol-2-yldiazenyl moiety. J Heterocycl Chem 2016;53:1820-6.

6. Hassan AS, Masoud DM, Sroor FM, Askar AA. Synthesis and biological evaluation of pyrazolo[1,5-a]pyrimidine-3-carboxamide as antimicrobial agents. Med Chem Res 2017;26:2909-10.

7. Kumar AK, Bodke YD, Lakra PS, Sambasivam G, Bhat KG. Design, synthesis and anti-cancer evaluation of a novel series of pyrazolo[1,5-a] pyrimidine substituted diamide derivatives. Med Chem Res 2017;26:714-44.

8. Rahmouni A, Souiei S, Belkacem MA, Romdhane A, Bouajila J, Ben Jannet $\mathrm{H}$, et al. Synthesis and biological evaluation of novel pyrazolopyrimidines derivatives as anticancer and anti-5-lipoxygenase agents. Bioorg Chem 2016;66:160-8.

9. Zhao M, Ren H, Chang J, Zhang D, Yang Y, He Y, et al. Design and synthesis of novel pyrazolo[1,5-a]pyrimidine derivatives bearing nitrogen mustard moiety and evaluation of their antitumor activity in vitro and in vivo. Eur J Med Chem 2016;119:183-96.

10. Li J, Zhao YF, Zhao XL, Yuan XY, Gong P. Synthesis and anti-tumor activities of novel pyrazolo[1,5-a]pyrimidines. Arch Pharm (Weinheim) 2006;339:593-7.

11. Ahmed OM, Mohamed MA, Ahmed RR, Ahmed SA. Synthesis and anti-tumor activities of some new pyridines and pyrazolo[1,5-a] pyrimidines. Eur J Med Chem 2009;44:3519-23.

12. Abdel-Aziz HA, Saleh TS, El-Zahabi HS. Facile synthesis and in-vitro antitumor activity of some pyrazolo[3,4-b]pyridines and pyrazolo[1,5-a]pyrimidines linked to a thiazolo[3,2-a]benzimidazole moiety. Arch Pharm (Weinheim) 2010;343:24-30.

13. Hassan AS, Hafez, TS, Osman SA, Ali MM. Synthesis and in vitro cytotoxic activity of novel pyrazolo[1,5-a]pyrimidines and related Schiff bases. Turk J Chem 2015;39:1102-13.

14. Ribeiro DA, Marques ME, Salvador DM. In vitro cytotoxic and nongenotoxic effects of gutta-percha solvents on mouse lymphoma cells by single cell gel (comet) assay. Braz Dent J 2006;17:228-32.

15. Shaikh IS, Zaheer Z, Mokale NS, Lokwani KD. Development of new pyrazole hybrids as antitubercular agents: Synthesis, biological evaluation and molecular docking study. Int $\mathrm{J}$ Pharm Pharm Sci 2017;9:50-6

16. Murulidharan V, Deepti CA, Raja S. A review on anti-inflammatory potential of substituted pyrazoline derivatives synthesised from chalcones. Int J Pharm Pharm Sci 2018;10:9.

17. Humphrey W, Dalke A, Schulten K. VMD-visual molecular dynamics. J Mol Graph 1996;14:33-5.

18. MTT Cell Proliferation Assay Instruction Guide - ATCC, VA, USA. Available from: http://www.atcc.org

19. Gerlier D, Thomasset N. Use of MTT colorimetric assay to measure cell activation. J Immunol Methods 1986;94:57-63.

20. Alley MC, Scudiero DA, Monks A, Czerwinski M, Shoemaker R $2^{\text {nd }}$, Boyd MR. Validation of an automated microculture tetrazolium assay (MTA) to assess growth and drug sensitivity of human tumor cell lines. Proc Am Assoc Cancer Res 1986;27:389. 